\title{
Rainer Eckertz Fünf Thesen zur Reform des Juristischen Studiums
}

1. Die von den juristischen Fakultäten eingeleitete Studienreform drobt unter der Decke einer allgemeinen Reformeuphorie die Effizienz des juristischen Lehrbetriebs unter Umgebung inhaltlicher und struktureller Reformen zu erböben.

Wer heute nach einer Reform des juristischen Studiums ruft, scheint offene Türen einzurennen. Daß man den sog. berechtigten Anliegen der Studenten Rechnung tragen müsse, ist längst ein beliebter Gemeinplatz von Politikern und Leitartiklern jeglicher Couleur geworden. Doch auch die Professoren scheinen diesmal zu spuren. Als die Studienreformkommission des Fakultätentags im Februar I 968 mit einem Studienreformplan an die Offentlichkeit trat, zeigte sich die Presse durchweg beeindruckt von der Entschiedenheit, mit der man hier Tabus zu brechen und alte Zöpfe abzuschneiden bereit zu sein schien. Indessen mußte schon damals stutzig werden, wer genauer hinsah: Als sei die Reform schon bestens im Gange, trug das Dokument den Titel »Münchener Beschlüsse zur Fortführung der Studienreform « (M. B.) $)^{1}$.

Man mag rätseln, worin der Anfang dieser Reform gesehen werden soll. Vielleicht in den "Baden-Badener Empfehlungen « des Fakultätentags vom Oktober 1967, in denen die Einführung einer »obligatorischen $Z$ wischenkontrolle mit bedingter Prüfung « nach 4 Semestern empfohlen wird, - vielleicht in dem schon seir längerem an den Juristischen Fakultäten verwirklichten *Tübinger Stufenplan «, nach dem als Voraussetzung der Zulassung zur Übung für Vorgerückte ein Anfängerschein zu erbringen ist, dessen Erwerb hinwiederum vom Nachweis der Teilnahme an einer Arbeitsgemeinschaft für Studienanfänger abhängig ist. Wenn aber all dies bereits als Studienreform gelten soll, muß es um die propagierte Radikalität der M. B. eigentümlich bestellt sein. In der Tat bekunden die M. B. in weiten Teilen einen geradezu beflissenen Konformismus². So wird den Professoren versichert, die von ihnen gehaltene Vorlesung sei die einzige Gewähr für eine wissenschaftliche Ausbildung und solle daher die zentrale Unterrichtsveranstaltung bleiben. Auch den Ubungen bisherigen Stils wird bescheinigt, sie hätten sich »insgesamt gesehen « bewährt. $Z$ war schlagen die M. B. einige marginale Veränderungen des Lehrbetriebs vor, in seinem Kern wollen sie ihn aber unverändert lassen. Der Horizont des Reformkonzepts der M. B. endet am Referendarexamen. Schon dessen organisatorischer Ablauf bleibt unangetastet. Die Problematik des oft beklagten Bruchs zwischen Universitärsstudium und praktischer Ausbildung wird schlicht verschwiegen.

\footnotetext{
1 Juristcnzeitung $68,223 \mathrm{f}$.

2 Th. Ramm, Juristenzeitung 68, 294 ff. (unter II, 2 u. 6) hat darauf hingewiesen, daß die M. B. - die Grenze respektieren, die Reformbestrebungen rvon innen gesetzt ist «.
} 
Worin also ist dann die vielgerühmte Radikalität der M. B. zu sehen? Sie brechen mit dem bisher für beide juristische Examen geltenden Vollständigkeitsprinzip und wollen den bisherigen Prüfungsstoff durch seine Aufteilung auf Kern- und Wahlfachgruppen reduzieren. Doch die Rigorosität, mit der die M. B. hierin verfahren, bleibt oberflächlich. Der vorhandene Fächerbestand wird rubriziert, ohne daß die Auswahl- und Einteilungskriterien expliziert werden. Die Stoffülle wird nicht durch inhaltliche Bestimmungen strukturiert, sondern nur formal begrenzt. $\mathrm{Zu}$ einer materiellen Neubestimmung der Stoffauswahl und -gewichtung sind indessen die M. B. auch gar nicht in der Lage, da sie niche die juristische Ausbildung in einen Bezug zur modernen Rechtswirklichkeit und der Funktion des Juristen in ihr setzen. Sie rekurrieren statt dessen auf die abstrakte Formel, Ziel der Ausbildung bleibe der $\gg$ fachlich geschulte, geistig selbständige und seiner Verantwortung bewußte junge Jurist《, wobei der Sinn dieser Atrribute offenbar ganz naiv als selbstverständlich unterstellt wird. Da es mithin an Maßstäben für eine inhaltliche Neubestimmung des Lehrstoffs fehlt, können die M. B. in ihrem Kernfächerkatalog auch nur die derzeit das Studium bestimmende Gewichtverteilung blindlings ratifizieren. Nach den M. B. bleibt es bei der Dominanz der traditionellen rechtsdogmatischen Fächer und der Vernachlässigung der Rechtsgebiete, in denen sich die modernen sozial-ökonomischen Entwicklungen niedergeschlagen haben.

Doch wo in Wahrheit nur der Prüfungsfächerkatalog der gegenwärtigen Studienwirklichkeit angepaßt wird, präsentiert sich der Offentlichkeit eine mit reformerischem Vokabular aufgeputzte Kulisse, von deren Schein sich nicht nur die sonst eher für die Studentenschaft Partei ergreifenden Teile der Presse, sondern auch manche wohlmeinende Professoren (bis in den Kreis der Studienreformkommission selbst hinein) haben trügen lassen. So soll die Stoffbeschränkung angeblich Raum für eine vertiefte wissenschaftliche Ausbildung schaffen, so soll durch das Wahlfachprinzip sich Studienfreiheit realisieren können. Gleichzeitig ist es aber das erklärte Ziel der M. B., wein wissenschaftliches Studium auch bei steigenden Studentenzahlen in angemessener Zeit zu ermöglichen*. Erinnert man sich hierbei daran, daß der Fakultätentag die Einführung der Zwangsexmatrikulation bereits in förmlichem Beschluß empfohlen hat, und bedenkt man, daß die traditionell orientierte Fächerauswahl der M. B. zugleich den optimalen Einsatz des vorhandenen Lehrpersonalbestandes bedeutet, so wird offensichtlich, daß hier eine Reform betrieben wird, die die Effizienz des bestehenden Ausbildungsapparats unter Umgehung inhaltlicher und struktureller Veränderungen steigern soll. Nähme man die proklamierten Ziele der Wissenschaftichkeit und Studienfreiheit wirklich ernst, so müßte man nach ihrer Realisierbarkeit im bestehenden Lehrbetrieb (große Vorlesung, Massenübung!) fragen. Da aber dieser Bezug nicht hergestellt wird, kann auch das Wahlfachprinzip nur als Mittel der Stoffreduzierung fungieren. $D a$ die juristischen Fakultäten zu einer inhaltlichen und strukturellen Reform nicht bereit sind, reagieren sie auf den zunehmenden Reformdruck regressiv durch Verkürzung der wissenschaftlichen Anforderungen an das Studium ${ }^{3}$.

Von seiten für die Studienreform engagierter Professoren pflegt derartiger Kritik entgegengehalten zu werden, daß durch die M. B. doch immerhin überhaupt erst einmal eine breite Debatte über die Studienreform in Gang gebracht

\footnotetext{
3 Der Studentenschaft wird also als Antwort auf ihre $\rightarrow$ Revolte eben die sich an den von den gegebenen Machtverhälenissen bestimmten Bedürfnissen orientierende Reform angeboten, die der Ausgangspunke der studentischen Kritik war.
} 
worden sei. In der Tat mußten viele Ordinarien erst ihr Fach durch die vorgeschlagene Reduzierung des Prüfungsfächerkatalogs bedroht sehen, um an der Studienreform Interesse zu finden. Dadurch aber, daB die in den M. B. entworfene Studienreform auf einer zu schmalen Basis ansetzt, ist die durch sie angestoßene Diskussion in fataler Weise determiniert ${ }^{4}$. Die bisherige Reformdebatte beschränkt sich fast ausschließlich auf die Erörterung des in den M. B. vorgeschlagenen Fächerkatalogs und das Entwerfen alternativer Fächerkataloge. Im Gerangel der Fachvertreter um einen Platz an der Sonne des Pflichtfächerkatalogs oder eine angemessene Entschädigung (die Lobby der Rechrsgeschichtler hat auf dem Fakultätentag vom 2r.6.68 die Einführung von Testaten für den Besuch der rechtsgeschichtlichen Vorlesung verlangt!), im munteren Erfinden immer neuer Wahlfachgruppen (die den Lehrern auch entlegener Gebiete Freude machen, ohne den Studenten weh zu tun) und in mannigfachen Spielchen mit den denkbaren Fächerkombinationen läßt sich eine schier endlose Diskussion bestreiten, die freilich nicht selten zur Farce mißrät. Hierüber wundern kann sich nur, wer den deutschen Ordinarius für ein in den Himmeln reiner Wissenschaft schwebendes, von irdischen Interessen gelöstes Wesen sieht. Da aber die bestehende Organisation des Lehrbetriebs den Ordinarien die Aufrechterhaltung ihrer überragenden Stellung in der Universität gewährleistet, da die Dominanz der traditionellen juristischen Disziplinen in der Lehre es den Ordinarien erspart, ihre Rolle in der modernen Demokratie zu problematisieren, und da das herrschende unpolitische Selbstverständnis der Rechtswissenschaft es den Ordinarien erleichtert, sich unauffällig mit den bestehenden gesellschaftlichen Verhältnissen zu identifizieren, steht zu befürchten, daß eine Studienreform, die mehr oder weniger reinen Ordinariengremien zur Beratung und Entscheidung überlassen bleibt, dazu führen wird, daß die unsere juristischen Fakultäten bedrängenden Reformforderungen so transponiert und eingedämmt werden, daß die bestehende Struktur des Lehrbetriebs, der etablierte Fächerbestand und das herrschende Selbstverständnis der Rechtswissenschaft nicht tangiert werden. Eine solche Entwidklung wäre durchaus vereinbar mit einer allgemeinen Reformeuphorie, der nun offenbar man auch unter den Ordinarien sich hinzugeben beginnt - freilich im Wege einer Autosuggestion.

2. Solange die Rechtswissenschaft nicht die politischen und sozioökonomischen Bedingtheiten des Rechts expliziert und sich nicht ibres Praxisbezugs durchgängig vergewissert, muß die Studienreform an der Desintegration des Lebrstoffs scheitern.

Den Gegnern einer Studienreform, die sich auf die Reduzierung des Prüfungsstoffs beschränkt, wird gern vorgeworfen, sie ignorierten das derzeit drängendste Problem einer Studienreform, die übermäßige Verlängerung der Studienzeit. Dem ist entgegenzuhalten, daß alle Versuche, der ausufernden Stoffülle durch eine bloße Beschränkung des Prüfungsfächerkatalogs Herr zu werden, nur zu Scheinlösungen führen können. Denn einmal ist eine formale Ausgrenzung weiter Rechtsgebiete aus dem Prüfungs- und damit fakrisch aus dem Lehrstoff, wie sie von den M. B. vorgeschlagen wird, letztlich undurchführbar, da Probleme, die formal abgewählten Gebieten zugehören, wegen ihres inhaltlichen Zusam-

4 Vgl. die Stellungnahme des FV Recheswissenschaft im VDS zu den M. B., Juristenzeitung Sonderheft zur Studienreform, Nov. 68, 6 ff. 
menhangs mit einem Kernfach unschwer von diesem her aufgerollt werden können. So könnten etwa mit Fug wirtschaftsrechtliche Fragen vom Schuldrecht her geprüft werden, oder das Tarifvertragsrecht von Art. 9 GG her ${ }^{5}$. Durch die auf dem Fakultätentag vom 21. 6.68 beschlossene Generalklausel, nach der auch die geschichtliche, theoretisch-methodologische, soziologische, ökonomische und politische Seite des Rechts zu den einzeinen Kernfächern gehört, steht eine Hintertür offen für beliebig ausdehnbare Ausfüge des Prüfers in das Gebiet der angeblich doch abwählbaren Grundlagenfächer. Zum anderen kann selbst das Ausklammern ganzer Bereiche aus dem Lernprozeß, wie es die M. B. vorsehen, angesichts der permanenten Expansion des Wissensstoffs nicht weiterhelfen. Denn da auch in den verbliebenen Bereichen das Wissen sich ständig vermehrt, kehrr über kurz oder lang das Problem der Stoffüberlastung wieder. Jede nur formale Begrenzung des Stoffs muß notwendigerweise an der zunehmenden Spezialisierung und den sich in der Entfaltung eines Problemfeldes eröffnenden Querverbindungen scheitern.

In Wahrheit ist aber auch gar nicht so sehr die Vermehrung des Wissensstoffs für die verlängerten Studienzeiten verantwortlich - einer Auswahl innerhalb der Gesamtmaterie bedurfte es selbstverständlich schon immer - als das Fehlen verbindlicher Kriterien für die Auswahl und Gewichtung des Lehr- und Prüfungsstoffs und die daraus resultierende Desorientierung des Studenten. Eine straffe Reglementierung des Studiengangs könnte diesen Orientierungsschwierigkeiten nicht abhelfen, da sie eine Folge des Desintegrationsprozesses sind, in dem sich gegenwärtig die Rechtsmaterie und die Rechtswissenschaft selbst befinden ${ }^{6}$. Dies mag zwar dem Studenten gewöhnlich nicht so recht bewußt werden, da er ja vorwiegend an Rechtsgebieten wie dem BGB geschult wird, in denen ein fester Lehrbestand überkommen ist, doch täuscht die relative dogmatische Konsistenz dieser Gebiete nur darüber hinweg, daß die Ankerpunkte ihrer Dogmatik (etwa die Privatautonomie) von der realen Entwicklung längst unterlaufen sind ${ }^{7}$. Ist so schon im traditionalen Lehrbestand der Boden brüchig geworden, so sind die neueren Rechtsgebiete wie Arbeits-, Wirtschafts-, Steuer- und Sozialrecht dogmatisch kaum konturiert. Dieses Manko wird in eigentümlicher Perversion wiederum als Grund dafür angeführt, daß diese Rechtsgebiete nicht in den Kernfächerkatalog aufgenommen werden, während sich aber doch dem Studenten erst hier die Perspektiven der heutigen Rechtswirklichkeit auftun könnten. So aber wird der Student immer noch im Geiste traditionell-liberaler Rechtsstaatlichkeit erzogen, obgleich das überkommene rechtsdogmatische Gebäude schon längst aus den Fugen geraten ist. Da jedoch diese Einsicht im herrschenden Selbstverständnis der Rechtswissenschaft unterdrückt wird, muß sich der Student mit den von der Lehre konservierten Fragmenten herumschlagen und es als subjektives Unvermögen erfahren, wenn diese sich nich zu einem Gesamtbild zusammenfügen lassen wollen.

Im Augenblick scheint es daher noch das beste Mittel einer Studienreform zu sein, durch Schaffung eines kritischen Bewußtseins der Studenten die Distanz zum Lehrstoff herzustellen, in der sich erst die Teilstücke ordnen und die Proportionen entzerren ließen. Da die Kategorien der herrschenden Rechtswissen-

5 Ramm, a.a.O. (unter II 3); R. Wiethölter, Stellungnahme zu den M. B., Juristenzeitung Sonderheft zur Studienreform, Nov. 68, g ff.

- Desintegration und Entrheoretisierung scheinen in der Gegenwart (zumindest) im Bereich der Sozialwissenschaften eine generelle Erscheinung zu sein. Dazu: W. Hofmann, Die Krise der Universität. In: ders., Universität, Ideologie, Gesellschaft, edition suhrkamp r g68, g ff.

7 Vgl. R. Wiechölter, a.a.O.; ders., Reche. In: Wissenschaft und Gesellschaft, Funkkolleg, Fischer Bücherei 1967, 215 ff., bes. 228 ff.; ders., Recheswissenschaft, Fischer Bücherei 1968. 
schaft nicht mehr realadäquat sind, läßt der theoretische Zusammenhang innerhalb der Rechtsmaterie sich zumindest vorerst nicht auf rechtsdogmatischem Wege wiedergewinnen, sondern nur in der Entfaltung des sozio-ökonomischen Zusammenhangs, durch den die in der juridischen Sphäre getrennten Glieder untergründig verbunden sind. Die sog. Grundlagenfächer müssen aus der musealen Edke, die ihnen der etablierte Lehrbetrieb zugedacht hat, befreit und in die fachimmanente Reflexion hereingeholt werden. Erst dann könnten die überkommenen Rechtsinstitute und Argumentationsmuster in ihrer historischen Relativität begriffen werden und zugleich eine Neustrukturierung der Rechtsmaterie in Angriff genommen werden. Ein von einer sozio-ökonomischen und rechtspolitischen Fragestellung angeleitetes Studium würde mit innerer Notwendigkeit auf die neueren sozialpolitisch gewichtigen Rechtsgebiete übergreifen. Nur auf diese Weise könnte auch einer rechtsdogmatischen Bewältigung dieser Materie der Weg gebahnt werden. Denn wenn diese Gebiete wie bisher einem pragmatisch lavierenden, parteiisch interessierten Verbandsexpertentum überlassen bleiben, wird es zu ihrer rechtstheoretischen Durchdringung nie kommen. - Das fundamentale, weil im derzeitigen Zustand der Rechtswissenschaft selbst begründete Problem der Desorientierung der Studenten läßt sich also nur auf der Grundlage eines langfristig zu konzipierenden Programms angehen. Die M. B. versprechen demgegenüber $z$ war rasche $H i l f e$, würden aber die eigentlich notwendige fundamentale Reform blockieren: Nicht nur weil sie das Bedürfnis nach einer Reform unterdrücken würden, sondern vor allem auch, weil das Studium nach dem Fächerkatalog der M. B. sowohl von den modernen, sozialpolitisch gewichrigen Rechtsgebieten als auch von den Grundlagenfächern abgeschnürt würde.

Eine auf die Restrukturierung der Lehrmaterie selbst abzielende Studienreform, wie sie hier gefordert wird, stößt freilich auf ein Hindernis, das nahezu unüberwindlich zu sein scheint: das unpolitische Selbstverständnis der herrschenden Rechtswissenschaft, die sich weigert, ihre sozio-ökonomischen Bedingtheiten zur Kennenis zu nehmen. Dem gegenüber gibt es derzeit nur die eine Hoffnung, daß die Eigendynaniik wissenschaftlichen Fragens sich gegen die in den juristischen Fakultäten institutionalisierten Beschränkungen der Rationalität durchzusetzen vermag. Von der Notwendigkeit, den bestehenden Wissensstoff gezielt zu vermitteln, geht ein $Z$ wang zur Reflexion auf den sozialen Kontext aus, innerhalb dessen sich die Lehr- und Lernprozesse vollziehen. Eine Didaktik der Rechtswissenschaft beginnt sich herauszubilden. Es wirft freilich ein bezeichnendes Licht auf das wissenschaftliche Niveau der Studienreformkommission des Fakultätentags, daß sie unter Didaktik hausbackene Ratschläge und Banalitäten ( $*$ Die Ubungen sollten so aufgebaut werden, daß sie zu einer systematischen Wiederholung des Lehrstoffs führen «) versteht. Im Gegensatz dazu wäre die didaktische Fragestellung von einer Analyse der bestehenden Lernprozesse über die Aufdeckung der Interdependenzen $z$ wischen dem Inhalt der Lehrprozesse und ihrem soziologischen Substrat weiterzutreiben zur Aufklärung des dialektischen Verhältnisses zwischen der Gesellschaft, in der sich das Recht konstituiert, und der Wissenschaft, die dieses Recht zu ihrem Gegenstand macht. Die Didaktik der Rechtswissenschaft ist heute eine jener $*$ Umschlagstellen $z$ wischen Theorie und Praxis $\kappa^{8}$, an denen nach der Einsicht von Habermas odie Selbstreflexion aufbriche «.

8 J. Habermas, Universitäc in der Demokratie - Demokratisierung der Universitär. In: Universitzätstage 1967, Berlin 1967,67 ff. (74). 
3. Eine Studienreform, die den Funktionswandel der juristischen Berufspraxis im modernen Interventionsstaat außer acht läßt, verhindert die Ubersetzung der Normen demokratisch-sozialer Rechtsstaatlichkeit in die Rechtswirklichkeit und paralysiert damit die Substanz der Rechtswissenschaft.

Für die Jurisprudenz gilt heute, was Karl Marx von der Philosphie sagte: man muß sie in die Armut ihres Selbstbewußtseins treiben. Marx' Einsicht, die Philosophie trage weder ihren Beginn noch ihr Ziel in sich, ist mutatis mutandis der Rechtswissenschaft vorzuhalten. Die Illusion, sie sei ein autonomer Bereich, verstellt der herrschenden Rechtslehre das Verständnis für die dem Juristen im modernen demokratischen Rechtsstaat zukommende Funktion ${ }^{9}$. Die Vorstellung eines über den politischen und sozialen Konflikten schwebenden Rechts nährt sich von einem Rechtsstaatsverständnis, das Staat und Gesellschaft trennt. Letzteres entstand bekanntlich selbst aus einer spezifischen historischen Interessenlage (der politischen Kapitulation des Bürgertums vor der Monarchie um den Preis einer Freigabe der ökonomischen Sphäre) und wird im heutigen Interventionsstaat täglich ad oculos widerlegt. Die moderne politische und ökonomische Wirklichkeit, in der sich öffentichrechtliche und privatrechtliche Sphäre ineinander verschränkt haben, kann in den Kategorien des traditionellen bürgerlichen Rechtsstaats, die von der Scheidung dieser beiden Sphären ausgehen, nicht mehr zutreffend beschrieben werden. Damit aber besteht die Gefahr, daß die Rechtswirklichkeit sich dem juristischen Zugriff überhaupt entzieht.

Franz Neumann hat bereits in der Zeit der Weimarer Republik gezeigt, wie sich im Ubergang vom Konkurrenz- zum organisierten Kapitalismus das System des liberalen Rechtsstaats auflöst ${ }^{10}$. Das formale (bestimmte und generelle) Gesetz des liberalen Rechtsstaats wird nach seinem Befund mehr und mehr verdrängt durch die Generalklausel einerseits, den individuellen Verwaltungsakt andererseits. Der Trend zur Entformalisierung des Rechts hat sich bis heute fortgesetzt ${ }^{11}$. Das auf rasche Veränderung angelegte Recht, mittels dessen die sozialen und ökonomischen Entwicklungen reguliert werden, ist rechtsdogmatisch kaum durchgebildet. - In der Praxis komme es daher immer weniger auf die Subsumtion vorgegebener Sachverhalte unter eine abstrakte Norm an, als auf die sachkundige, akrive soziale Gestaltung 1. Damit wird aber dem berufsspezifischen Monopol der Juristen, der Subsumtionskunst, der Boden entzogen. Der Schwerpunkt der erforderlichen Qualifikationen verschiebt sich auf die Fähigkeit zur wissenschaftichen Analyse der zur Entscheidung anstehenden sozialen Sachverhalte und die Beherrschung von Entscheidungstechniken. Im Bereich der Rechrsprechung hat der Bedeutungsschwund des formalen Gesetzes zur Anerkennung einer richterlichen Rechtsfortbildung geführt, mit der Hinnahme vorlegaler Standards aber auch das Problem der Rationalität der Entscheidungsfindung aufgeworfen ${ }^{13}$.

- G. Wittkämper, Die Rechtswissenschaft angesidhts sozialwissenschaftlicher Beratung der Politik, Juristenzeitung $68,449 \mathrm{ff}$.

$10 \mathrm{Fr}$. Neumann, Der Funktionswandel des Gesetzes im Recht der bürgerlichen Gesellscaft. In: ders., Demokratischer und autoritärer Staat, F. a. M. 1967, 31 ff.

11 W. Hofmann, Die Krise des Staates und das Recht. In: Kritische Justiz, $1968 \mathrm{H}$. I, 1 ff.

12 Dies zeigt an Hand einer Analyse der Funktion des Juristen in der heutigen Bildungsverwaltung I. Richter, Skizzen zum Verhälenis von Juristenausbildung und Sozialwissenschaft, Berlin 1967, unveröffentlicht.

13 S. zum ganzen Problemkreis G. Bechmann/B. Moldenhauer/E. Osborg/H. Rottleuthner, Zur politischen Reorganisation des Jurastudiums, in: Universität und Widerstand F. 2. M. 1968, 130 \&. 
Das juristische Studium indessen bereitet auf diese Probleme in keiner Weise vor. Die gegenwärtige juristische Ausbildung ist noch weithin der spärbürgerlich-liberalen Vorstellungswelt verhaftet. Ihr Leirbild ist im Grunde immer noch der Privarrechrskonflikte entscheidende, in »denkendem Gehorsam « der Weisheit des Gesetzes vertrauende Richter. In den Ubungen und den meisten Vorlesungen wird eine rein rechtslogisch verfahrende Subsumtionskunst an von der Lebenswirklichkeir abstrahierten Fällen eingeübt. Da der Student fast nur an den aus dem liberalen Rechtsstaat überkommenen Materien ausgebildet wird, stößt der Lehrbetrieb sich nicht an den für die moderne Rechtswirklichkeit spezifischen Schwierigkeiten. $\mathrm{Da}$ die Rechtswissenschaft überdies durch die Rückständigkeit ihrer Grundlagenfächer von den Entwicklungen innerhalb der Sozialwissenschaften isoliert ist, drohe der eingefahrenen Lehre auch von daher keine Gefahr. Die Universität hat so immer mehr die Züge eines von der Rechtswirklichkeir abgekapselten Reservats klassischer Jurisprudenz angenommen, das mit der *Praxis nur noch an den Spitzen, im Dialog professoraler und höchstrichterlicher Olympier, kommuniziert ${ }^{14}$.

Das unpolitische Selbstverständnis der herrschenden Rechrswissenschaft beschirmt diese vor der erschredkenden Einsicht in die Diskrepanz zwischen Universitätsjuristerei und Rechtspraxis und verdeckt eine heimliche Paralyse der Rechtsstaatlichkeit. Denn wenn die Jurisprudenz zur Bewältigung der Rechtswirklichkeit nicht mehr in der Lage ist, bedeutet das ja nicht, daß die Gesellschaft in Anarchie versinkr, wie ein sich absolut setzender Gesetzespositivismus glauben mag, sondern nur, daß die sozialen Prozesse auf andere als rechtliche Weise normiert werden ${ }^{15}$. Wo der Jurist verdrängt wird, rüdkt der Sozialtechniker nach. Je realinadäquater die juristischen Kategorien werden, desto undurchsichtiger werden die von den herrschenden gesellschaftlichen Bedürfnissen determinierten sozioökonomischen Funktionsabläufe. Angesichts der Perfektion einer gleichsam naturgesetzlich verfestigten und technokratisch verwalteten Zivilsation ${ }^{18}$ kann der Jurist nur noch ratlos beiseite stehen. Der Jurisprudenz käme in dieser sozialtechnisch manipulierbaren Gesellschaft nur noch eine ästhetische Funktion zu: Sie hätte, indem ihr scheinbar arbeitsteilig die Entscheidung sozialer Konfliktsfälle zugeschoben würde, die realen Verantwortlichkeiten zu verdecken. Die Rechrswissenschaft wird sich zur Ästhetisierung der bestehenden gesellschaftlichen Ordnung um so leichter mißbrauchen lassen, als sie in vermeintlicher Autonomie sich an von der sozioökonomischen Entwidklung überholten Ordnungsbildern orientiert. Eine Jurisprudenz, die sich nicht mehr absolut setzte, begriffe es als ihre Aufgabe, das in das Grundgesetz eingegangene Versprechen eines demokratischen und sozialen Rechtsstaats dadurch einzulösen, daß sie die überkommenen Rechtsinstitute in den veränderten sozialen Kontext sübersetzt«. Eine juristische Ausbildung, die sich von diesem Ziel her verstünde, dürfte weder von den modernen, sozialpolitisch erheblichen Rechtsgebieten noch von den Entwicklungen in den Sozialwissenschaften absehen. Solange aber die Rechtslehre, weil sie auf ihre Autonomie vertraut, vor der Aufgabe versagt, die Juristen zu Obersetzern der demokratisch-sozialen Rechtsstaatlichkeit in die gesellschaftliche Wirklichkeit auszubilden, droht der Rechtswissenschaft eben diese

\footnotetext{
14 Dazu aus der Perspektive der ridhterlichen Praxis die (leider völlig theorielose) Kritik des Pseudonymus Xaver Berra, Im Paragraphenturm, Neuwied a. Rh. 1966.

15 Dazu Wittkämper, a.a.O.

10 Sie wird als bercits real unterstellt von $\mathrm{H}$. Shelsky, Einsamkeit und Freiheit, rde 1963 , I86 ff. 225. Kritisch dazu J. Habermas an vielen Stellen, z. B. Dogmatismus, Vernunt und Enrscheidung - $Z_{u}$ Theoric und Praxis in der verwissenschaflichen Zilivisazion. In: ders., Theorie und Praxis, 2. Aufl. Neuwied 2. Rh. 1967, 23 If.
} 
Wirklichkeit und mit ihr das Recht selbst zu entgleiten. Sofern in der gegenwärtigen Diskussion um die Studienreform diese Gefahr nicht bewußt wird, kann es in der Tat dazu kommen, daß die Rechtslehre einer nur noch nach quasi natürlichen Sachgesetzlichkeiten ablaufenden, den Kriterien der Gerechtigkeit verschlossenen Gesellschaft auf einer eigens hierzu bereitgestellten Bühne das Bild des Rechtsstaats vorgaukelt.

4. Eine Studienreform, die nicht das Ziel einer individuellen Emanzipation der Studenten in sich aufnimmt, muß an deren Unfäbigkeit zur Reflexion des Lebrstoffs scheitern.

Die hier gegen die gegenwärtig von den juristischen Fakultäten betriebene Studienreform erhobene Anklage gründet auf dem Vorwurf eines dreifachen Verrats: Eine solche Reform verrät nicht nur die Rechtswissenschaft und mit ihr den demokratischen Rechtsstaat, sondern indem sie um einer bloß formalen Straffung des Studiums willen den Studenten zum Verrat an der Wissenschaft und am demokratischen Rechtsstaat anhält, verrät sie auch das Interesse des Studenten an seiner Emanzipation. Die Verfechter dieses Reformkonzepts können dabei freilich auf die Bereitschaft der Studenten zum Selbstverrat bauen. Ihr liebstes Argument ist, die große Mehrheit der Studenten wolle ja nichts anderes als ein straff geleitetes Studium ${ }^{17}$. - Der tatsächlich vorhandene Wunsch vieler Studenten, unter der Last eines diffundierenden Lehrstoffs in einem durchreglementierten, streng kontrollierten Apparat Halt zu finden, wäre aber zu begreifen als das Ergebnis eines Ausbildungssystems, in dem die eigentliche Triebkraft aller Bildungsprozesse, das Interesse des Lemenden an Mündigkeit, nicht geweckt sondern unterdrückt wird. Das die gegenwärtige Studienwirklichkeit - besonders bei den Juristen - bestimmende Motiv ist die Erwartung der Studenten, für die langjährige Hinnahme der Inferioritäten eines Schülerdaseins einmal durch einen Platz in den vorderen Rängen unserer Gesellschaftsordnung honoriert zu werden. Einem nur noch vom Karriere-Interesse bestimmten Studenten muß in der Tat am möglichst reibungslosen Durchlauf durch die Universität und an der Ausmerzung irritierender Relikte von Studienfreiheit gelegen sein. Indessen bringt die bloße Glättung eines in seiner Struktur unveränderten Lehrbetriebs dem Studenten nur scheinbar eine Entlastung. Mit der Ausräumung der Widerstände, an denen sich abmühend der Student sich in seine Wissenschaft einarbeiten würde, wird dem Studium die Sache selbst entzogen. An den entgleitenden Wissensstoff muß der Student nunmehr mit $Z$ wang herangebracht werden.

Eine Studienreform, die diesen Zusammenhang nicht reflektiert, gerät in einen circulus vitiosus. Der bestehende Lehrbetrieb vermag es nicht, wissenschaftliches Interesse zu wecken. Zwar liebt es mancher Professor, vor seinen Hörern ein intellektuelles Feuerwerk abzubrennen, zwar müssen die Studenten sich in Ubungen und Seminaren zuweilen wissenschaftlich gebärden, doch täuschen diese Komödien nur darüber hinweg, daß im bestehenden Ausbildungssystem eine Tendenz objektiv verankert ist, den Lehrstoff zu entproblematisieren. Das Wissen wird in Massenvorlesungen und -übungen, in denen der Student auf eine bloß rezeptive Rolle beschränkt ist, durch die Autoritätsfigur des Ordinarius vermit-

17 Gegen dieses pseudodemokratische Argument läßt sich nur innerhalb einer Treue-VerratDialekrik angehen. Zu dieser aus religionsphilosophischer Siór K. Heinrioh, Versuch über die Schwierigkeit nein zu sagen, F. 2. M 1964. Der Begriff des Oberserzens (vgl. unsere 3. These) steht in diesem Denken an zentraler Stelle. 
telt und ist damit vor Kritik weitgehend abgesichert. Der psychische Druck, der vom Examen ausgeht, führt dazu, daß der Student in tatsächlicher oder auch vermeintlicher Zeitnor den Stoff nicht denkend durchdringt, sondern mechanisch einpaukt. Das florierende Geschäft der Repetitoren bezeugt dies deutlich genug. Eine solche Ausbildung bringt Juristen hervor, die statt den Stoff rational zu beherrschen, an ihn psychisch fixiert sind ${ }^{18}$. - Der juristische Lehrstoff trägt aber, wie wir zu zeigen versucht haben, seine Vernunft immer weniger in sich. Gegenüber der auseinanderfallenden Wissensmaterie kann sich nur noch behaupten, wer ein reflekriert-distanziertes Verhältnis zu ihr gewonnen hat. Da aber im etablierten Lehrbetrieb die ganz große Mehrzahl der Studenten an der Reflexion des Lehrstoffs verhindert ist, gerät die Studienreform in den Sog des Desintegrationsprozesses der Rechtswissenschaft selbst. Die Orientierung, die in der Lehrmaterie selbst die Studenten nicht finden können, suchen sie in der Anlehnung an die Autorität der Lehrenden. $\mathrm{Da}$ aber auch die Sicherheit, die diese ihnen geben können, nur trügerisch ist, grassiert heute im Studium eine immer ungreifbarer werdende Unsicherheit, die ihrerseits das Bedürfnis nach administrativer Meisterung des sich aus sich selbst heraus zerstörenden Lehrbetriebs immer unabweisbarer werden läßt.

Die heute drängendste praktische Frage ist daher, wie diese regressive Tendenz in der sich abzeichnenden »Studienreform « überwunden werden kann. Es gibt nur einen Weg - den der Bewußtwerdung. Sofern die Rechtswissenschaft den Prozeß wissenschaftlichen Fragens nicht willkürlich abbricht, wird sie durch die Sache selbst gezwungen sein, sich auf ihre Praxis zu besinnen. Es ist freilich recht unwahrscheinlich, daß die an seniler Demens leidende Rechtslehre, die derzeit die Universitäten beherrscht, von sich aus den beschwerlichen Gang der fachimmanenten Reflexion antreten wird. Hier könnte aber einer kritisch gewordenen studentischen Minderheit eine maieutische Funktion zufallen. Die im Namen einer Kritischen Universität gebildeten studentischen Gruppen hätten die Ergebnisse ihrer Arbeit in den etablierten Lehrbetrieb hineinzutragen und der dort bisher vor einem gläubigen Publikum ihren Schaum schlagenden ${ }^{19}$ Rechtslehre einen Widerstand entgegenzusetzen, der sie zwingt sich zu konturieren ${ }^{20}$. Diese Arbeitsgruppen könnten sich aus dem in der Studentenschaft zur Zeit noch vorhandenen oppositionellen Potential rekrutieren, in dem die Einsicht zu verbreiten wäre, daß die in unserer Gesellschaftsordnung eingenistete Herrschaft durch eine sich kritisch verstehende Rechtswissenschaft wohl wirksamer bekämptt werden könnte als durch noch so eifriges Fahnenschwingen. Eine studentische Gruppenarbeit, die sich politisch versteht, die Diskrepanz zwischen Rechtslehre und Rechtswirklichkeit begrifflich zu fassen sich anstrengt und sich dem bestehenden Lehrbetrieb in der Absicht zuwendet, ihn zu verändern, wäre zugleich auch die beste Eigenhilfe in der gegenwärtigen Studiennot. Der Lehrstoff ließe sich zwanglos strukturieren, wenn er um seiner handlungsorientierenden Funktion willen aufgearbeitet würde 21 .

Daher ist unsere letzte These:

Wenn die regressive Tendenz in der sich gegenwärtig abzeichnenden Studien-

18 Wern D. Claessens, Bemerkungen zur Soziologie der deurschen Universitzät, in: Universitärstage 1967 , Berlin 1967,54 f. ( 56$)$, es als die eigentliche Funktion der Universität in der hochkomplexen Gesellschaft bestimme hac, ^Reflexivität herzustellen und aufrechezuerhalten, so kann man die derzeitige juristische Ausbildung geradezu als " Antistudium * charakterisieren.

19 -Sc九uma ist bei K. Heinrich eine Metapher für Struktur- und Identitärslosigkeit.

:o $\mathrm{Zu}$ einem derartigen Versuch Frankfurter Studenten vgl. G. Bechmann/B. Moldenhauer/ E. Osborg/H. Rottleuthner, a.a.O. $130 \mathrm{ff}$., $149 \mathrm{ff}$.

91 Vgl. O. Negt. Soziologische Phantasie und exemplarisches Lernen, F. a. M. 1968. 
reform überwunden werden soll, muß der bestehende Lebrbetrieb durch die Konfrontation mit einer studentischen Minderbeit, die in kritischer Absicht den Lehrstoff aufarbeitet, gezwungen werden zum Bewußtsein seiner selbst zu gelangen?2.

22 Ober eine solche Konfrontation an der Frankfurter Juristischen Fakultät vgl. den Kormmentar von $H$. Schacht auf der nächsten Seite. 